\title{
The Euler numbers and recursive properties of Dirichlet $L$-functions
}

\author{
Yiwei Hou' and Shimeng Shen ${ }^{2 *}$ (D)
}

"Correspondence: millieshen28@163.com

${ }^{2}$ School of Mathematics, Northwest University, Xi'an, China

Full list of author information is available at the end of the article

\begin{abstract}
The aim of this paper is using an elementary method and the properties of the Bernoulli polynomials to establish a close relationship between the Euler numbers of the second kind $E_{n}^{*}$ and the Dirichlet $L$-function $L(s, \chi)$. At the same time, we also prove a new congruence for the Euler numbers $E_{n}$. That is, for any prime $p \equiv 1 \bmod 8$, we have $E_{\frac{p-3}{2}} \equiv 0 \bmod p$. As an application of our result, we give a new recursive formula for one kind of Dirichlet L-functions.
\end{abstract}

MSC: 11B83; 11B37

Keywords: Euler numbers; Euler numbers of the second kind; Bernoulli polynomials; Dirichlet L-function; Identity; Congruence

\section{Introduction}

For any integer $n \geq 0$ and real number $0 \leq x<1$, the Euler polynomials $E_{n}(x)$ (see $[1,2]$ and [3]) and the Bernoulli polynomials $B_{n}(x)$ (see [2, 4] and [5]) are defined by the coefficients of the power series

$$
\frac{2 e^{z x}}{e^{z}+1}=\sum_{n=0}^{\infty} \frac{E_{n}(x)}{n !} \cdot z^{n} \quad \text { and } \quad \frac{z \cdot e^{z x}}{e^{z}-1}=\sum_{n=0}^{\infty} \frac{B_{n}(x)}{n !} \cdot z^{n} .
$$

When $x=0, E_{n}=E_{n}(0)$ is called the $n$th Euler number, $B_{n}=B_{n}(0)$ is called the $n$th Bernoulli number. For example, the initial values of $E_{n}$ and $B_{n}$ are $E_{0}=1, E_{1}=-\frac{1}{2}, E_{2}=0, E_{3}=\frac{1}{4}, E_{4}=$ $0, E_{5}=-\frac{1}{2}, E_{6}=0, \ldots ; B_{0}=1, B_{1}=-\frac{1}{2}, B_{2}=\frac{1}{6}, B_{3}=0, B_{4}=-\frac{1}{30}, B_{5}=0, B_{6}=\frac{1}{42}, \ldots$.

The Euler numbers of the second kind $E_{n}^{*}$ (see $[2,6,7]$ and [8]) are also defined by the coefficients of the power series

$$
\frac{2}{e^{z}+e^{-z}}=\sum_{n=0}^{\infty} \frac{E_{n}^{*}}{n !} \cdot z^{n}=\sum_{n=0}^{\infty} \frac{E_{2 n}^{*}}{(2 n) !} \cdot z^{2 n},
$$

where $E_{0}^{*}=1, E_{2}=-1, E_{4}^{*}=5, E_{6}^{*}=-61$, and $E_{2 i+1}^{*}=0$ for all integers $i \geq 0$.

It is clear that $E_{n}^{*}=2^{n} \cdot E_{n}\left(\frac{1}{2}\right)$. These polynomials and numbers arise in many combinatorial and number theory contexts. As for the elementary properties of these sequences, various authors have studied them and obtained many interesting results. For example, W. Zhang [9] obtained some combinational identities. As an application of the result in

(c) The Author(s) 2018. This article is distributed under the terms of the Creative Commons Attribution 4.0 International License (http://creativecommons.org/licenses/by/4.0/), which permits unrestricted use, distribution, and reproduction in any medium, provided you give appropriate credit to the original author(s) and the source, provide a link to the Creative Commons license, and indicate if changes were made. 
[9], he proved that for any prime $p$, one has the congruence

$$
(-1)^{\frac{p-1}{2}} E_{p-1}^{*} \equiv\left\{\begin{array}{lll}
0 & \bmod p & \text { if } p \equiv 1 \bmod 4 \\
-2 & \bmod p & \text { if } p \equiv 3 \bmod 4
\end{array}\right.
$$

Richard K. Guy [10] (see problem B45 and [11]) proposed the following two problems: Is it true that for any prime $p \equiv 1 \bmod 8, p \nmid E_{\frac{p-1}{2}}^{*}$ ? Is the same true for $p \equiv 5 \bmod 8$ ?

G. Liu [6] solved the second problem by an elementary method. Later, W. Zhang and $\mathrm{Z}$. Xu [7] solved the above two problems completely. In fact, they proved the following general conclusion: For any prime $p \equiv 1 \bmod 4$ and positive integer $\alpha$, one has the congruence

$$
p^{\alpha} \nmid E_{\phi\left(p^{\alpha}\right) / 2}^{*},
$$

where $\phi(n)$ denotes the Euler function.

Recently, J. Zhao and Z. Chen [12] proved the following conclusion: For any positive integers $n$ and $k \geq 2$, one has the identity

$$
\sum_{a_{1}+a_{2}+\cdots+a_{k}=n} \frac{E_{a_{1}}}{\left(a_{1}\right) !} \cdot \frac{E_{a_{2}}}{\left(a_{2}\right) !} \cdots \frac{E_{a_{k}}}{\left(a_{k}\right) !}=\frac{2^{k-1}}{(k-1) !} \cdot \frac{1}{n !} \sum_{i=0}^{k-1} C(k-1, i) E_{n+k-1-i},
$$

where the sequence $\{C(k, i)\}$ is defined as follows: For any positive integer $k$ and integers $0 \leq i \leq k$, we define $C(k, 0)=1, C(k, k)=k$ ! and

$$
C(k+1, i+1)=C(k, i+1)+(k+1) C(k, i), \quad \text { for all } 0 \leq i<k,
$$

provided $C(k, i)=0$, if $i>k$.

As corollaries of this result, J. Zhao and Z. Chen [12] also obtained the following results: For any odd prime $p$, one has the congruences

$$
E_{p+1} \equiv 0(\bmod p), \quad 2 E_{p} \equiv 1(\bmod p) \quad \text { and } \quad E_{p-1} \equiv-1(\bmod p) .
$$

T. Kim et al. (see [2,13-17] and [18]) also obtained many interesting identities related to $E_{n}$ and $E_{n}^{*}$. Especially in [19], T. Kim also proved a series of important conclusions involving Euler numbers and polynomials associated with zeta functions.

In this paper, we will use elementary methods and the properties of the Bernoulli numbers to establish a close relationship between the Euler numbers of the second kind $E_{n}^{*}$ and the Dirichlet $L$-function $L(s, \chi)$. Meanwhile, we will also prove a new congruence for the Euler numbers $E_{n}$. That is to say, we will prove the following several facts.

Theorem 1 For any positive integer $n$, we have the identity

$$
E_{2 n}^{*}=(-1)^{n} \cdot \frac{2^{2 n+2} \cdot(2 n) !}{\pi^{2 n+1}} \cdot L\left(2 n+1, \chi_{4}\right)
$$


where $\chi_{4}$ denotes the non-principal character mod 4 , and $L\left(s, \chi_{4}\right)$ denotes the Dirichlet $L$ function corresponding to $\chi_{4}$. In fact, we have

$$
\chi_{4}(n)= \begin{cases}0 & \text { if } 2 \mid n ; \\ (-1)^{\frac{n-1}{2}} & \text { if } 2 \nmid n .\end{cases}
$$

Theorem 2 For any positive integer $n$, we have the identity

$$
E_{2 n-1}=-\frac{\left(2^{2 n}-1\right)}{n} \cdot B_{2 n}
$$

From Theorems 1 and 2 we may immediately deduce the following:

Corollary 1 For any positive integer $n$, we have the recursive formula

$$
\sum_{k=0}^{n} \frac{(-1)^{k}}{(2 n-2 k) !} \cdot \frac{4^{k}}{\pi^{2 k+1}} \cdot L\left(2 k+1, \chi_{4}\right)=0
$$

where $L\left(1, \chi_{4}\right)=\frac{\pi}{4}$.

Corollary 2 Let $p$ be a prime with $p \equiv 1 \bmod 8$, then we have the congruence

$$
E_{\frac{p-3}{2}} \equiv 0 \bmod p
$$

Corollary 3 For any positive integer $n$ with $(n, 3)=1$, we have the congruence

$$
E_{2 n-1} \equiv 1 \bmod 3 .
$$

Corollary 4 For any positive integer $n$ with $(n, 5)=1$, we have the congruence

$$
E_{4 n-1} \equiv-1 \bmod 5
$$

Corollary 5 For any positive integer $n$ with $(n, 7)=1$, we have the congruence

$$
E_{6 n-1} \equiv 3 \bmod 7
$$

Corollary 6 For any positive integer $n$ with $(n, 11)=1$, we have

$$
E_{10 n-1} \equiv 1 \bmod 11
$$

From Theorem 2 we can also deduce the following identities:

$$
L\left(1, \chi_{4}\right)=\frac{\pi}{4} ; \quad L\left(3, \chi_{4}\right)=\frac{\pi^{3}}{32} ; \quad L\left(5, \chi_{4}\right)=\frac{5 \cdot \pi^{5}}{1536} \quad \text { and } \quad L\left(7, \chi_{4}\right)=\frac{61 \cdot \pi^{7}}{184,320}
$$

Some notes Since $E_{n}$ is not necessarily an integer, it can still be written as $E_{n}=\frac{H_{n}}{K_{n}}$ with $\left(H_{n}, K_{n}\right)=1$. So $E_{n} \equiv 0 \bmod p$ in this paper implies that $p \mid H_{n}$ while $p \nmid K_{n}$.

For a prime $p \equiv 5 \bmod 8$, whether $E_{\frac{p-3}{2}} \equiv 0 \bmod p$ is true is an interesting open problem. 


\section{Several simple lemmas}

In this section, we will give two simple lemmas. Hereafter, we may use facts from number theory and the properties of the Bernoulli numbers, all of which can be found in [4]. Thus we will not repeat them here.

Lemma 1 For any positive integer $n$ and real number $x$, we have the identity

$$
2^{n} \cdot B_{n}(x)=\sum_{k=0}^{n}\left(\begin{array}{l}
n \\
k
\end{array}\right) E_{k} \cdot B_{n-k}(2 x)=\sum_{k=0}^{n}\left(\begin{array}{l}
n \\
k
\end{array}\right) E_{k}(2 x) \cdot B_{n-k} .
$$

Proof First from the definitions of the Euler numbers and Bernoulli polynomials we have

$$
\begin{aligned}
\frac{2 z e^{2 x z}}{e^{2 z}-1} & =\frac{2}{e^{z}+1} \cdot \frac{z e^{2 z x}}{e^{z}-1}=\left(\sum_{n=0}^{\infty} \frac{E_{n}}{n !} \cdot z^{n}\right) \cdot\left(\sum_{n=0}^{\infty} \frac{B_{n}(2 x)}{n !} \cdot z^{n}\right) \\
& =\sum_{n=0}^{\infty} \frac{1}{n !}\left(\sum_{k=0}^{n}\left(\begin{array}{l}
n \\
k
\end{array}\right) E_{k} \cdot B_{n-k}(2 x)\right) \cdot z^{n} .
\end{aligned}
$$

On the other hand, from the definition of the Bernoulli polynomials and the Euler polynomials, we also have

$$
\frac{2 z e^{2 x z}}{e^{2 z}-1}=\sum_{n=0}^{\infty} \frac{B_{n}(x)}{n !} \cdot(2 z)^{n}=\sum_{n=0}^{\infty} \frac{2^{n} \cdot B_{n}(x)}{n !} \cdot z^{n}
$$

and

$$
\begin{aligned}
\frac{2 z e^{2 x z}}{e^{2 z}-1} & =\frac{2 e^{2 x z}}{e^{z}+1} \cdot \frac{z}{e^{z}-1}=\left(\sum_{n=0}^{\infty} \frac{E_{n}(2 x)}{n !} \cdot z^{n}\right)\left(\sum_{n=0}^{\infty} \frac{B_{n}}{n !} \cdot z^{n}\right) \\
& =\sum_{n=0}^{\infty} \frac{1}{n !}\left(\sum_{k=0}^{n}\left(\begin{array}{l}
n \\
k
\end{array}\right) E_{k}(2 x) \cdot B_{n-k}\right) \cdot z^{n} .
\end{aligned}
$$

Combining (2)-(4) and comparing the coefficients of the power series, we have the identity

$$
2^{n} \cdot B_{n}(x)=\sum_{k=0}^{n}\left(\begin{array}{l}
n \\
k
\end{array}\right) E_{k} \cdot B_{n-k}(2 x)=\sum_{k=0}^{n}\left(\begin{array}{l}
n \\
k
\end{array}\right) E_{k}(2 x) \cdot B_{n-k} .
$$

This proves Lemma 1.

Lemma 2 For any positive integer $n$, we have the identity

$$
B_{2 n+1}\left(\frac{1}{4}\right)=(-1)^{n+1} \frac{2(2 n+1) !}{(2 \pi)^{2 n+1}} L\left(2 n+1, \chi_{4}\right),
$$

where $\chi_{4}$ denotes the non-principal character mod 4.

Proof For any real number $0<x<1$, from [4, Theorem 12.19] we have

$$
B_{2 n+1}(x)=(-1)^{n+1} \frac{2(2 n+1) !}{(2 \pi)^{2 n+1}} \sum_{k=1}^{\infty} \frac{\sin (2 \pi k x)}{k^{2 n+1}} .
$$


Taking $x=\frac{1}{4}$ in (5), we have

$$
\begin{aligned}
B_{2 n+1}\left(\frac{1}{4}\right) & =(-1)^{n+1} \frac{2(2 n+1) !}{(2 \pi)^{2 n+1}} \sum_{k=1}^{\infty} \frac{\sin \left(\frac{2 \pi k}{4}\right)}{k^{2 n+1}} \\
& =(-1)^{n+1} \frac{2(2 n+1) !}{(2 \pi)^{2 n+1}} \sum_{k=1}^{\infty} \frac{\sin \left(\frac{2 \pi(2 k-1)}{4}\right)}{(2 k-1)^{2 n+1}} \\
& =(-1)^{n+1} \frac{2(2 n+1) !}{(2 \pi)^{2 n+1}} \sum_{k=1}^{\infty} \frac{(-1)^{k-1}}{(2 k-1)^{2 n+1}}=(-1)^{n+1} \frac{2(2 n+1) !}{(2 \pi)^{2 n+1}} \sum_{k=1}^{\infty} \frac{\chi_{4}(k)}{k^{2 n+1}} \\
& =(-1)^{n+1} \frac{2(2 n+1) !}{(2 \pi)^{2 n+1}} L\left(2 n+1, \chi_{4}\right) .
\end{aligned}
$$

This proves Lemma 2.

\section{Proofs of the theorems}

In this section, we will complete the proofs of our theorems. First, we prove Theorem 1. For any positive integer $m$, taking $x=\frac{1}{4}$ and $n=2 m+1$ in Lemma 1 , and noting that $B_{1}=-\frac{1}{2}$ and $B_{2 i+1}=E_{2 i+1}\left(\frac{1}{2}\right)=0$ for all integers $i \geq 1$, we have

$$
\begin{aligned}
2^{2 m+1} \cdot B_{2 m+1}\left(\frac{1}{4}\right) & =\sum_{k=0}^{2 m+1}\left(\begin{array}{c}
2 m+1 \\
k
\end{array}\right) E_{k}\left(\frac{1}{2}\right) B_{2 m+1-k} \\
& =\left(\begin{array}{c}
2 m+1 \\
2 m
\end{array}\right) E_{2 m}\left(\frac{1}{2}\right) \cdot B_{1}=-\frac{2 m+1}{2} E_{2 m}\left(\frac{1}{2}\right)=-\frac{2 m+1}{2^{2 m+1}} E_{2 m}^{*} .
\end{aligned}
$$

From (6) and Lemma 2 we have

$$
2^{2 m+1} \cdot(-1)^{m+1} \frac{2(2 m+1) !}{(2 \pi)^{2 m+1}} L\left(2 m+1, \chi_{4}\right)=-\frac{2 m+1}{2^{2 m+1}} E_{2 m}^{*}
$$

or

$$
E_{2 m}^{*}=(-1)^{m} \cdot \frac{2^{2 m+2} \cdot(2 m) !}{\pi^{2 m+1}} \cdot L\left(2 m+1, \chi_{4}\right) .
$$

This proves Theorem 1 .

Taking $x=0, n=2 m$ and $m \geq 1$ in Lemma 1 , we have

$$
2^{2 m} \cdot B_{2 m}(0)=2^{2 m} \cdot B_{2 m}=\sum_{k=0}^{2 m}\left(\begin{array}{c}
2 m \\
k
\end{array}\right) E_{k} \cdot B_{2 m-k}(0)=\sum_{k=0}^{2 m}\left(\begin{array}{c}
2 m \\
k
\end{array}\right) E_{k} \cdot B_{2 m-k} .
$$

Note that $B_{2 i+1}=0$ for all $i \geq 1$, and $B_{1}=-\frac{1}{2}, E_{0}=1$ and $E_{2 i}=0$ for all $i \geq 1$. From (7) we have

$$
2^{2 m} \cdot B_{2 m}=\left(\begin{array}{c}
2 m \\
0
\end{array}\right) E_{0} \cdot B_{2 m}+\left(\begin{array}{c}
2 m \\
2 m-1
\end{array}\right) E_{2 m-1} \cdot B_{1}=B_{2 m}-m \cdot E_{2 m-1},
$$

which implies

$$
E_{2 m-1}=-\frac{2^{2 m}-1}{m} \cdot B_{2 m}
$$

This proves Theorem 2. 
Now we prove Corollary 1 . For any positive integer $n$, note that the power series

$$
\frac{e^{z}+e^{-z}}{2}=\sum_{n=0}^{\infty} \frac{z^{2 n}}{(2 n) !}
$$

from the definition of $E_{2 n}^{*}$ satisfies the identity

$$
1=\left(\sum_{n=0}^{\infty} \frac{1}{(2 n) !} \cdot z^{2 n}\right) \cdot\left(\sum_{n=0}^{\infty} \frac{E_{2 n}^{*}}{(2 n) !} \cdot z^{2 n}\right)=\sum_{n=0}^{\infty}\left(\sum_{k=0}^{n} \frac{E_{2 k}^{*}}{(2 k) !} \cdot \frac{1}{(2 n-2 k) !}\right) \cdot z^{2 n}
$$

That is, for any positive integer $n$, we have the identity

$$
\sum_{k=0}^{n} \frac{E_{2 k}^{*}}{(2 k) !} \cdot \frac{1}{(2 n-2 k) !}=0
$$

Combining (8) and Theorem 1, we may immediately deduce the identity

$$
\sum_{k=0}^{n} \frac{(-1)^{k}}{(2 n-2 k) !} \cdot \frac{4^{k}}{\pi^{2 k+1}} \cdot L\left(2 k+1, \chi_{4}\right)=0
$$

This proves Corollary 1.

To prove other corollaries, taking a prime $p=4 k+1$ and $n=\frac{p-1}{4}$ in Theorem 2 , we have

$$
E_{\frac{p-3}{2}}=-\frac{4}{p-1} \cdot\left(2^{\frac{p-1}{2}}-1\right) \cdot B_{\frac{p-1}{2}} .
$$

From Euler's criterion (see [4, Theorem 9.2]) we have

$$
2^{\frac{p-1}{2}} \equiv\left(\frac{2}{p}\right) \equiv(-1)^{\frac{p^{2}-1}{8}} \equiv \begin{cases}1 \bmod p & \text { if } p \equiv 1 \bmod 8 \\ -1 \bmod p & \text { if } p \equiv 5 \bmod 8\end{cases}
$$

where $\left(\frac{*}{p}\right)$ denotes the Legendre's symbol $\bmod p$.

For the Bernoulli numbers $B_{2 n}$, we also have

$$
B_{2 n}=I_{n}-\sum_{p-1 \mid 2 n} \frac{1}{p}
$$

where $I_{n}$ is an integer and the sum is over all primes $p$ such that $p-1$ divides $2 n$.

In fact, formula (11) was discovered in 1840 by von Staudt and Clausen (independently); see [4, Exercises for Chap. 12].

Now in (11), we let $2 n=\frac{p-1}{2}$ and $B_{2 n}=\frac{U_{2 n}}{V_{2 n}}$, where $U_{2 n}$ and $V_{2 n}$ are two integers with $\left(U_{2 n}, V_{2 n}\right)=1$. Since $p-1 \nmid \frac{p-1}{2}$, from $(11)$ we know that $\left(V_{2 n}, p\right)=1$ and $\left(2^{\frac{p-1}{2}}-1\right) \cdot B_{\frac{p-1}{2}}$ is an integer.

If $p \equiv 1 \bmod 8$, then from (9), (10) and (11) we have the congruence

$$
E_{\frac{p-3}{2}}=-\frac{4}{p-1} \cdot\left(2^{\frac{p-1}{2}}-1\right) \cdot B_{\frac{p-1}{2}} \equiv 0 \bmod p .
$$

This proves Corollary 2. 
Corollaries 3-6 can also be easily deduced from Theorem 2 and the method used when proving Corollary 2.

This completes the proofs of all our results.

If $p \equiv 5 \bmod 8$, then we have $2^{\frac{p-1}{2}} \equiv-1 \bmod p$ and $p \nmid\left(2^{\frac{p-1}{2}}-1\right)$. So in this case, whether one has $E_{\frac{p-3}{2}} \equiv 0 \bmod p$ remains an open problem.

\section{Acknowledgements}

The authors wish to express their gratitude to the editors and the reviewers for their helpful comments.

\section{Funding}

This work is supported by the N.S.F. (11771351) of P.R. China.

\section{Competing interests}

The authors declare that there are no conflicts of interest regarding the publication of this paper.

Authors' contributions

All authors have equally contributed to this work. All authors read and approved the final manuscript.

\section{Author details}

${ }^{1}$ Department of Fundamental Science, Hebei Finance University, Baoding, China. ${ }^{2}$ School of Mathematics, Northwest University, Xi'an, China.

\section{Publisher's Note}

Springer Nature remains neutral with regard to jurisdictional claims in published maps and institutional affiliations.

Received: 19 September 2018 Accepted: 16 October 2018 Published online: 25 October 2018

\section{References}

1. Knuth, D.E., Buckholtz, T.J.: Computation of tangent, Euler, and Bernoulli numbers. Math. Comput. 21, $663-688$ (1967)

2. Kim, D.S., Kim, T.: Some $p$-adic integrals on $\mathbb{Z}_{p}$ associated with trigonometric functions. Russ. J. Math. Phys. 25, 300-308 (2018)

3. Liu, G.D.: Identities and congruences involving higher-order Euler-Bernoulli numbers and polynomials. Fibonacci Q. 39, 279-284 (2001)

4. Apostol, T.M.: Introduction to Analytic Number Theory. Springer, New York (1976)

5. Kim, D.S., Park, K.H.: Identities of symmetry for Bernoulli polynomials arising from quotients of Volkenborn integrals invariant under $S_{3}$. Appl. Math. Comput. 219, 5096-5104 (2013)

6. Liu, G.D.: The solution of problem for Euler numbers. Acta Math. Sin. 47, 825-828 (2004)

7. Zhang, W.P., Xu, Z.F.: On a conjecture of the Euler numbers. J. Number Theory 127, 283-291 (2007)

8. Zhang, W.P.: On the several identities of Euler numbers. J. Northwest Univ. Nat. Sci. 22, 17-20 (1992) (Chinese)

9. Zhang, W.P.: Some identities involving the Euler and the central factorial numbers. Fibonacci Q. 36, 154-157 (1998)

10. Guy, R.K.: Unsolved Problems in Number Theory, 2nd edn. Springer, New York (1994)

11. Powell, B.J.: Advanced problem 6325. Am. Math. Mon. 87, 836 (1980)

12. Zhao, J.H., Chen, Z.Y.: Some symmetric identities involving Fubini polynomials and Euler numbers. Symmetry 10, 303 (2018)

13. Kim, D.S., Kim, T.: Some symmetric identities for the higher-order $q$-Euler polynomials related to symmetry group $S_{3}$ arising from $p$-adic $q$-fermionic integrals on $\mathbb{Z}_{p}$. Filomat 30, 1717-1721 (2016)

14. Kim, T.: Symmetry of power sum polynomials and multivariate fermionic $p$-adic invariant integral on $\mathbb{Z}_{p}$. Russ. J. Math. Phys. 16, 93-96 (2009)

15. Kim, T., Kim, D.S., Jang, G.W.: A note on degenerate Fubini polynomials. Proc. Jangjeon Math. Soc. 20, $521-531$ (2017)

16. Kim, T., Kim, D.S.: An identity of symmetry for the degenerate Frobenius-Euler polynomials. Math. Slovaca 68 , 239-243 (2018)

17. Kim, T., Kim, S.D., Jang, G.W., Kwon, J.: Symmetric identities for Fubini polynomials. Symmetry 10, 219 (2018)

18. Rim, S.H., Jeong, J.H., Lee, S.J., Moon, E.J., Jin, J.H.: On the symmetric properties for the generalized twisted Genocchi polynomials. Ars Comb. 105, 267-272 (2012)

19. Kim, T.: Euler numbers and polynomials associated with zeta functions. Abstr. Appl. Anal. 2008, Article ID 581582 (2008)

20. Simsek, Y.: Identities on the Changhee numbers and Apostol-type Daehee polynomials. Adv. Stud. Contemp. Math. 27, 199-212(2017) 